\title{
Efficient input and output fiber coupling to a photonic crystal waveguide
}

\author{
Paul E. Barclay, Kartik Srinivasan, Matthew Borselli, and Oskar Painter \\ Department of Applied Physics, California Institute of Technology, Pasadena, California 91125
}

Received November 3, 2003

\begin{abstract}
The efficiency of evanescent coupling between a silica optical fiber taper and a silicon photonic crystal waveguide is studied. A high-reflectivity mirror on the end of the photonic crystal waveguide is used to recollect, in the backward-propagating fiber mode, the optical power that is initially coupled into the photonic crystal waveguide. An outcoupled power in the backward-propagating fiber mode of $88 \%$ of the input power is measured, corresponding to a lower bound on the coupler efficiency of 94\%. (C) 2004 Optical Society of America OCIS codes: $\quad 060.1810,130.3120$.
\end{abstract}

Evanescent coupling between optical fiber tapers ${ }^{1}$ and photonic crystal waveguides ${ }^{2,3}$ (PCWGs) allows highly efficient and mode-selective interfacing between fiber optics and planar photonic crystal (PC) devices. Circumventing the intrinsic spatial and refractive-index mismatch of fiber to PCWG end-fire coupling, ${ }^{4}$ this coupling scheme takes advantage of the strong dispersion and undercut geometry inherent to PC membrane structures to allow efficient power transfer between modes of a PCWG and a fiber taper. The coherent interaction over the length of the coupling region between phase-matched modes of each waveguide manifests in nearly complete resonant power transfer between waveguides, and varying the position of the fiber taper makes it possible to tune the coupling strength and phase-matching frequency, allowing the dispersive and spatial properties of PCWG modes to be efficiently probed. ${ }^{5}$

In previous work ${ }^{2,5}$ the coupling efficiency of this technique was studied by analyzing the signal transmitted through the fiber taper as a function of wavelength and fiber taper position. Although transmission measurements are sufficient to infer the efficiency of this coupling scheme, a measurement of power coupled into and then back out of a PCWG is of interest since it allows the lower bound of the input-output coupling efficiency to be directly established. By studying light coupled into a PCWG, reflected by an end mirror at the PCWG termination, and then coupled into the backward-propagating fiber mode, we show in this Letter that this coupling scheme has near-unity efficiency.

Figure 1(a) shows an illustration of the fiber taper evanescent coupling scheme. A fiber taper, formed by simultaneously heating and stretching a standard single-mode fiber until it has a minimum diameter of roughly $1 \mu \mathrm{m}$, is positioned above and parallel to an undercut planar PCWG. The small diameter of the fiber taper allows the evanescent tail of its fundamental mode to interact with the PCWG, and the undercut geometry of the PCWG prevents the fiber taper from radiating into the $\mathrm{PC}$ substrate. A schematic detailing the optical path within the fiber taper and the PCWG is shown in Fig. 1(b). To spectrally characterize the taper-PCWG coupler, an external-cavity swept-wavelength source with a wavelength range of
1565-1625 nm was connected to the fiber taper input by means of a $3-\mathrm{dB}$ coupler. At the fiber taper output a photodetector $\left(\mathrm{D}_{\mathrm{T}}\right)$ was connected to measure the transmitted power past the taper-PCWG coupling region. An additional photodetector $\left(D_{R}\right)$ was connected to the second 3-dB coupler input to measure the light reflected by the PCWG.

The PCWGs used in this work were fabricated from a silicon-on-insulator wafer. The airholes were patterned in the 300-nm-thick silicon layer with standard electron-beam lithography and dry etching techniques, after which the suspended membrane structure was created by selective removal of the $2-\mu \mathrm{m}$-thick underlying silicon dioxide layer with a hydrofluoric acid wet etch. To form the PCWGs a grade in hole radius was introduced along the transverse direction of a compressed square lattice of airholes ${ }^{6}$ as shown in Figs. 2(a) and 2(b). Coupling in this case occurs between the fundamental linearly polarized fiber
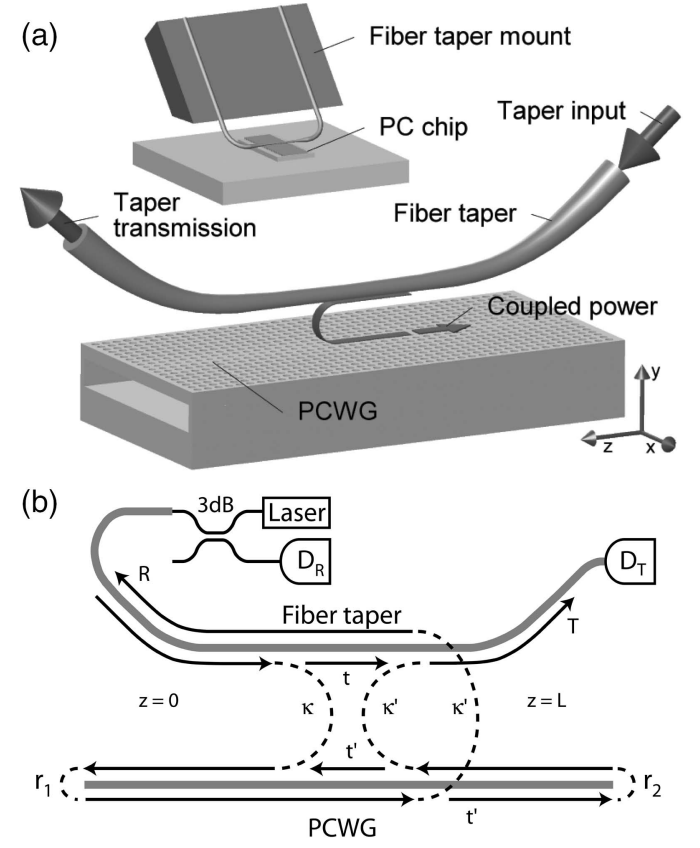

Fig. 1. (a) Schematic of the coupling scheme. (b) Illustration of the contradirectional coupling process and the feedback within the PCWG caused by the reflectivities $r_{1,2}$ of the waveguide terminations. 


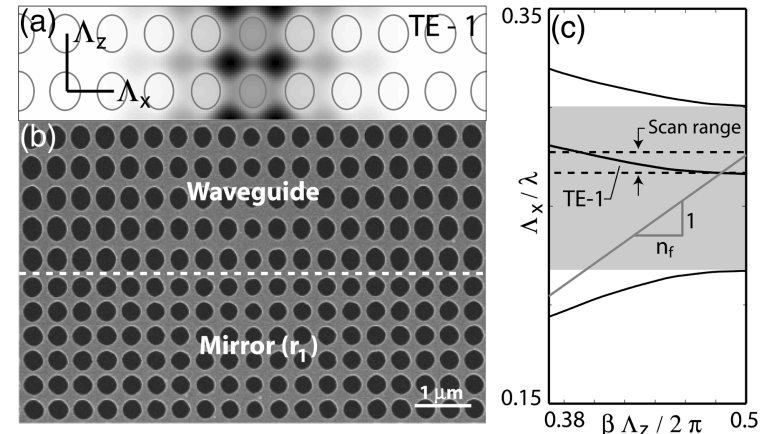

Fig. 2. (a) Waveguide geometry and finite-difference timedomain calculated magnetic field profile $\left(B_{y}\right)$ of the TE-1 mode. (b) Scanning electron microscopy image of the high $\left(r_{1}\right)$ reflectivity waveguide termination. The PCWG has a transverse lattice constant $\Lambda_{x}=415 \mathrm{~nm}$, a longitudinal lattice constant $\Lambda_{z}=536 \mathrm{~nm}$, and length $L=200 \Lambda_{z}$. (c) Dispersion of the TE-1 PCWG mode. Also shown are the mirror termination band edges for momentum along the waveguide axis $(\hat{z})$. The corresponding partial bandgap of the mirror region is indicated by the shaded region.

mode and the TE-1 PCWG mode, the field profile of which is shown in Fig. 2(a). This PCWG mode has a negative group velocity, resulting in contradirectional coupling as depicted in Fig. 1, and was designed to be integrated with recently demonstrated ${ }^{7}$ high- $Q$ cavities formed with a similar grading in hole radius.

In previous studies of evanescent coupling to such PCWGs ${ }^{5}$ the waveguide termination consisted of a $2-\mu \mathrm{m}$ region of undercut silicon, followed by an $8-\mu \mathrm{m}$ length of nonundercut silicon on insulator and a final air interface. The interference from multiple reflections at the three interfaces within the waveguide termination resulted in a highly wavelength-dependent reflection coefficient, making quantitative analysis of signals reflected by the PCWG difficult. Here, the waveguide terminations were engineered to have either high or low broadband reflectivities. On one end of the PCWG, shown in Fig. 2(b), a PC mirror with high modal reflectivity $\left(r_{1}\right)$ for the TE-1 PCWG mode was used. This high-reflectivity end mirror was formed by removal of the lattice compression of the waveguide while maintaining the transverse grade in hole radius. The change in lattice compression results in the TE-1 PCWG mode lying within the partial bandgap ${ }^{6}$ of the high-reflectivity end mirror section [Fig. 2(c)] while the transverse grade in hole radius reduces diffraction loss within the mirror. On the opposite end of the PCWG a poor reflector with low reflectivity $\left(r_{2}\right)$ was realized by removal (over several lattice constants) of the transverse grade in hole radius while keeping the central waveguide hole radius fixed, resulting in a loss of transverse waveguiding within the end mirror section and allowing the TE-1 PCWG mode to diffract into the unguided bulk modes of the PC.

In the absence of reflections caused by the PCWG termination (i.e., $r_{1,2}=0$ ) the fiber-PCWG junction can be characterized by

$$
\left[\begin{array}{c}
a_{F}^{+}(L) \\
a_{\mathrm{PC}}^{-}(0)
\end{array}\right]=\left[\begin{array}{cc}
t & \kappa^{\prime} \\
\kappa & t^{\prime}
\end{array}\right]\left[\begin{array}{c}
a_{F}^{+}(0) \\
a_{\mathrm{PC}}^{-}(L)
\end{array}\right]
$$

where $\kappa$ and $\kappa^{\prime}$ are coupling coefficients; $t$ and $t^{\prime}$ are transmission coefficients; and $a_{F}^{+}(z)$ and $a_{\mathrm{PC}}^{-}(z)$ are the amplitudes of the forward-propagating fundamental fiber taper mode and the backward-propagating TE-1 PCWG mode, respectively. The coupling region extends along the $z$ axis, with $z=0$ corresponding to the input and $z=L$ to the output of the coupler. As illustrated in Fig. 1(b), nonzero $r_{1,2}$ introduce feedback into the system. This allows input light coupled from the forward-propagating fundamental fiber taper mode into the TE-1 PCWG mode to be partially reflected by the PCWG termination and subsequently coupled to the backward-propagating fundamental fiber taper mode. In the presence of this feedback within the PCWG, the normalized reflected and transmitted powers in the fiber taper are given by ${ }^{8}$

$$
\begin{aligned}
& T=\left|a_{F}^{+}(L)\right|^{2}=\left|t+\frac{\kappa \kappa^{\prime} t^{\prime} r_{1} r_{2}}{1-r_{1} t^{\prime} r_{2} t^{\prime}}\right|^{2}, \\
& R=\left|a_{F}^{-}(0)\right|^{2}=\left|\frac{\kappa \kappa^{\prime} r_{1}}{1-r_{1} t^{\prime} r_{2} t^{\prime}}\right|^{2},
\end{aligned}
$$

for $a_{F}^{+}(0)=1$ and $a_{\mathrm{PC}}^{-}(L)=0$. By measuring $T$ and $R$ and considering Eqs. (2) and (3), we can determine the efficiency of the fiber-PCWG coupling as measured by $\left|\kappa \kappa^{\prime}\right|$.

In the measurements presented here a fiber taper with $2-\mu \mathrm{m}$ diameter was used to probe the PCWG of Fig. 2(b). Figures 3(a) and 3(b) show $T$ and $R$ as a function of wavelength when the taper is aligned with the PCWG at a height of $g=0.20 \mu \mathrm{m}$ and indicate that the phase-matched wavelength is $\lambda \sim 1598 \pm$ $5 \mathrm{~nm}$. As described in Ref. 5, coupling to the guided TE- 1 mode of the PCWG was confirmed by study of the coupling dependence upon polarization, lateral taper displacement, and fiber taper diameter. Figure 3(c) shows $T$ and $R$ at the resonant wavelength of the PCWG nearest the phase-matching condition [the minima and maxima in $T(\lambda)$ and $R(\lambda)$ ] as a function of taper height $g$ above the PCWG. Included in Fig. 3(c) is the off-resonant (away from phase matching) transmission, $T_{\text {off }}$, through the fiber. A maximum normalized reflected power $R_{\max } \sim 0.88$ was measured at a height of $g \sim 0.25 \mu \mathrm{m}$, where the corresponding transmission was $T<0.01$. As can be seen in the wavelength scans of Fig. 3(a), at this taper height the Fabry-Perot resonances caused by multiple reflections from the end mirrors of the PCWG are suppressed for wavelengths within the coupler bandwidth due to strong coupling to the fiber taper. Ignoring for the moment the effects of multiple reflections in Eq. (3), the maximum optical power coupling efficiency is then $\left|\kappa \kappa^{\prime}\right|=\sqrt{R_{\max }} /\left|r_{1}\right|$, where the square-root dependence upon $R_{\max }$ is a result of the light passing through the coupler twice in returning to the backward-propagating fiber mode. Assuming the high-reflectivity mirror is perfect (unity reflection), for the measured $R_{\max }=0.88$ this implies $94 \%$ coupling of optical power from taper to PC waveguide (and vice versa).

By further comparison of $T(g)$ and $R(g)$ with the model given above, this time including feedback 

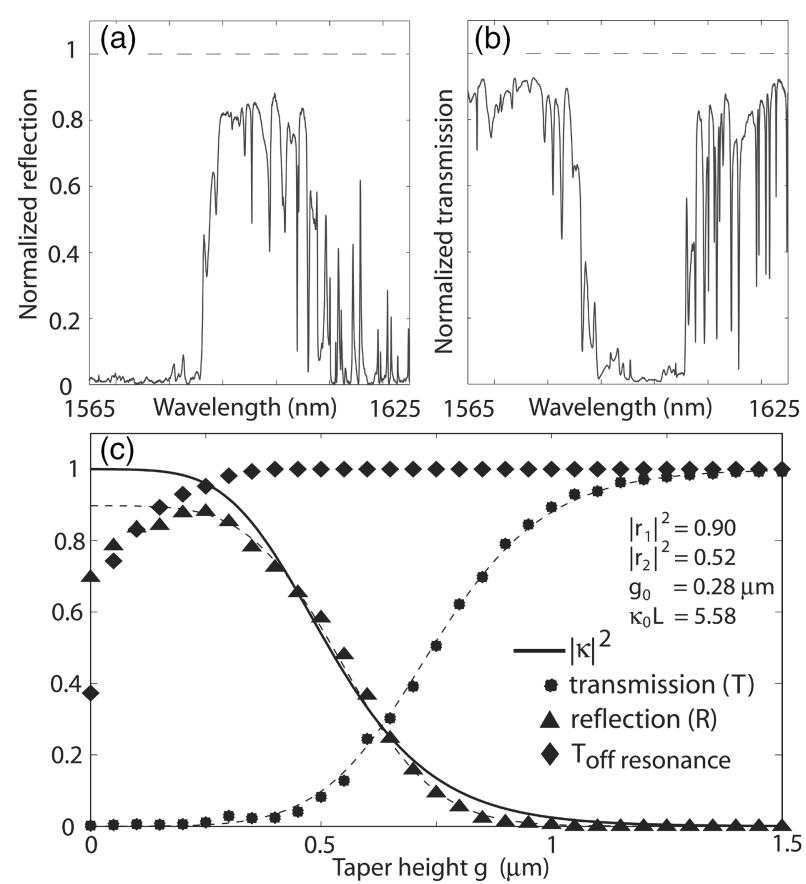

Fig. 3. (a) Reflection and (b) transmission of the fiber taper as a function of wavelength for a taper height of $0.20 \mu \mathrm{m}$. Both signals were normalized to the taper transmission with the PCWG absent. (c) Measured taper transmission minimum $(\lambda \sim 1600 \mathrm{~nm})$, reflection maximum $(\lambda \sim$ $1600 \mathrm{~nm})$, and off-resonant transmission $(\lambda \sim 1565 \mathrm{~nm})$ as a function of taper height. Also shown are fits to the data, and the resulting predicted coupler efficiency, $|\kappa|^{2}$. Asymmetry in the fiber taper loss about the coupling region was taken into account by repeating the measurements with the direction of propagation through the taper and the orientation of the PCWG sample reversed; the geometric mean of the values obtained from the two orientations takes any asymmetry into account.

within the PCWG, we find that the high-reflectivity PCWG end mirror is imperfect and that $R_{\max }$ is in fact limited by mirror reflectivity, not the efficiency of the coupling junction. For this comparison we take the elements of the coupling matrix to satisfy the relations $|t|^{2}+|\kappa|^{2}=1, \kappa^{\prime}=\kappa$, and $t^{\prime}=t^{*}$ of an ideal (lossless) coupling junction. ${ }^{8}$ For the phase-matched contradirectional coupling considered here, the dependence of $\kappa$ on $g$ can be approximated by $|\kappa(g)|=\tanh \left[\kappa_{\perp, 0} \exp \left(-g / g_{0}\right) L\right],{ }^{9}$ where $\kappa_{\perp, 0}$ and $g_{0}$ are constants. Substituting these relations into Eqs. (2) and (3), we can fit $T$ and $R$ to the experimental data with $r_{1,2}, \kappa_{\perp, 0}$, and $g_{0}$ as free parameters. ${ }^{10}$ Waveguide loss can also be included in the model; however, it is found to be small compared with the mirror loss and will be studied in detail elsewhere. Figure 3(c) shows the fits to the data for $g \geq 0.20 \mu \mathrm{m}$; for $g<0.20 \mu \mathrm{m}$ nonresonant scattering loss is no longer negligible and the ideal coupling junction model breaks down. Note that this scattering loss is observed to affect the reflected signal at a smaller taper-PCWG gap than for the transmitted signal; $T_{\text {off }}$ begins to decrease for $g \leq 0.30 \mu \mathrm{m}$, whereas $R$ begins to decrease for $g \leq 0.20 \mu \mathrm{m}$. This indicates that, within the coupler bandwidth, $1-T_{\text {off }}$ is an overestimate of the amount of power scattered into radiation modes. From the fits in Fig. 3(c) the PCWG end mirror reflectivities are estimated to be $\left|r_{1}\right|^{2}=0.90$ and $\left|r_{2}\right|^{2}=0.52$, and the optical power coupling efficiency $\left(|\kappa|^{2}\right)$ occurring at $R_{\max }(g=0.25 \mu \mathrm{m})$ is approximately $97 \%$.

In conclusion, these results indicate that this coupling scheme allows highly efficient input and output coupling between photonic crystal devices and fiber tapers and is promising for efficient probing of future integrated planar PC devices.

K. Srinivasan thanks the Hertz Foundation and M. Borselli thanks the Moore Foundation for financial support. P. Barclay's e-mail address is pbarclay@ caltech.edu.

\section{References}

1. J. Knight, G. Cheung, F. Jacques, and T. Birks, Opt. Lett. 22, 1129 (1997).

2. P. E. Barclay, K. Srinivasan, M. Borselli, and O. Painter, IEE Electron. Lett. 39, 842 (2003).

3. W. Kuang, C. Kim, A. Stapleton, and J. O'Brien, Opt. Lett. 27, 1604 (2002).

4. M. Notomi, A. Shinya, K. Yamada, J. Takahashi, C. Takahashi, and I. Yokohama, IEEE J. Quantum Electron. 38, 736 (2002).

5. P. E. Barclay, K. Srinivasan, M. Borselli, and O. Painter, arXiv.org e-Print archive, quant-ph/0308070, August 12, 2003, http://arXiv.org/quant-ph/0308070.

6. P. E. Barclay, K. Srinivasan, and O. Painter, J. Opt. Soc. Am. B 20, 2274 (2003).

7. K. Srinivasan, P. E. Barclay, M. Borselli, and O. Painter, arXiv.org e-Print archive, quant-ph/0309190, September 25, 2003, http://arXiv.org/quant-ph/ 0309190.

8. H. A. Haus, Waves and Fields in Optoelectronics, Prentice-Hall Series in Solid State Physical Electronics (Prentice-Hall, Englewood Cliffs, N.J., 1984), Chap. 3, pp. 59-71.

9. P. Yeh and H. F. Taylor, Appl. Opt. 19, 2848 (1980).

10. We take $r_{1} t^{\prime} r_{2} t^{\prime} /\left|r_{1} t^{\prime} r_{2} t^{\prime}\right|=1$, corresponding to a resonant condition within the PCWG. 